\title{
An Algorithm for Investigating the Structure of Material Surfaces
}

\author{
M. Toman, P. Procházka
}

The aim of this paper is to summarize the algorithm and the experience that have been achieved in the investigation of grain structure of surfaces of certain materials, particularly from samples of gold.

The main parts of the algorithm to be discussed are:

1. acquisition of input data,

2. localization of grain region,

3. representation of grain size,

4. representation of outputs (postprocessing).

Keywords: investigation of structure of material surfaces, transformation of images, Gaussian low-frequency filter, local equalization, probability distribution of grain size.

\section{Introduction}

Data describing the grain structure and shape of a surface of a material sample are obtained using STM (a type of microscope), see Section 3. STM has been selected on basis of experience gathered from many publications on this topic. For example, in scanning of gold islands grown on MoS2 surfaces STM proved that this approach is powerful, [7], [9-10].

The input data for this algorithm are taken from STM in the form of a gray-scale image. The aims of the algorithm are:

- to localize boundaries between adjacent grains and to localize grain regions (for one grain there is one and only one region),

- to depict a graph of the found probability distribution of grain size.

The localization of grain boundaries may be applied to an investigation of any materials with a surface structure similar to a grain structure. The studied materials have to fulfill basically the condition to be conductive, as STM is based on the principle of a tunnel current between the microscope and the sample. For the localization, segmentation techniques are used in a wide range of classical image analysis problems. For example, the task of segmenting cell nuclei from cytoplasm is a classical image analysis problem, which may prove to be crucial to the development of successful systems that automate the analysis for detection of cancer of the cervix, [4-6].

As soon as the boundaries between grains are localized, they may be used for various calculations. Within the scope of the algorithm described in this paper, the data describing the boundaries are used for calculating the probability distribution of grain size. The gray-scale images, providing information on the grain structure of the sample surface, are often very noisy and have to be smoothed (i.e., their quality should be improved) with the use of various filters. Unfortunately, the use of such filters leads to the partial loss of important detailed information. This partial loss of information and high demands on calculations lead to an inaccurate resulting description of the grain boundaries. For this reason, the data representing the grain boundaries are used for calculating the probability distribution of the investigated parameters of the grain structure. If the quality of the grey- -scale images were improved, the results of the algorithm described in the following text would be enhanced.

The grain size is expressed by the grain radius (it is assumed that grains can be approximated by a sphere). The outputs of the algorithm are represented by a graph.

The gray-scale images suffer from technical deficiencies of the microscope, but they are not relevant for the calculations.

The images of the grain structure of gold samples are used for illustrating the algorithm.

\section{Transformations applied to the gray-scale images}

This section describes important transformations, such as the square median filter, Gaussian low-frequency filter and local equalization, which are widely applied to gray-scale images. Further information about transformations and digital image processing in general is presented in many books, e.g., [1-2].

\subsection{Square median filter}

When applying a square median filter (further in the text referred as the "square median filter") to the image, one has to carry out the following steps for each point (pixel) in the image:

- get intensities in the square neighborhood (with the given size) of the point,

- find the median value of the intensities,

- store this median value to the buffer at the appropriate coordinate that belongs to the point.

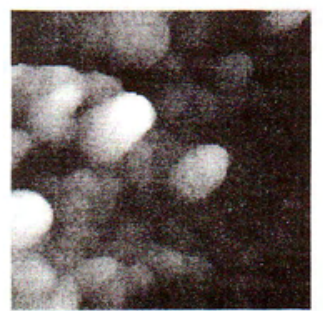

Fig. 1: Image after applying the square median filter 
The entire buffer will represent the image after transformation.

The result of the application of the square median filter is seen in Fig. 1.

\subsection{Gaussian low-frequency filter}

The application of the Gaussian filter to the gray-scale image involves convolution of the image with the matrix filled with the values given by the following function:

$$
\mathrm{e}^{((-0.5 / \text { (value of diffusion } \cdot \text { value of diffusion }))((x \cdot x)+(y \cdot y)))},
$$

where $[x, y]$ indicate indices to the matrix and $[0,0]$ is the center of the matrix.

The Gaussian filter removes the high frequencies formed due to mistakes in scanning. The result of the application of the Gaussian filter is illustrated in Fig. 2.

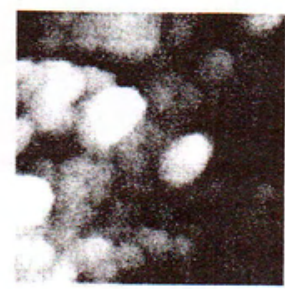

Fig. 2: Image after applying the Gaussian low-frequency filter

\section{3 (Local) equalization of the histogram of the gray-scale image}
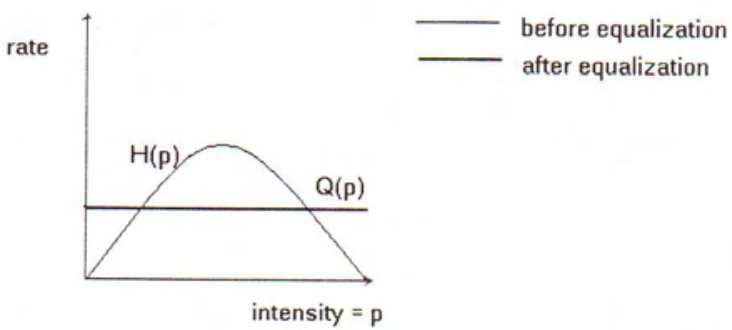

Fig. 3: Histogram of the image before and after applying equalization

Local equalization emphasizes local intensity peaks, local minima (boundaries between grains) and unifies changes of brightness in the whole image.

If $h \cdot v$ is the size of neighborhood of a given point for local equalization (or the image size for global equalization), then we substitute the intensity of the center of neighborhood (or each intensity ranged from 0 to 255 for global equalization) by such an intensity obtained from the following formula:

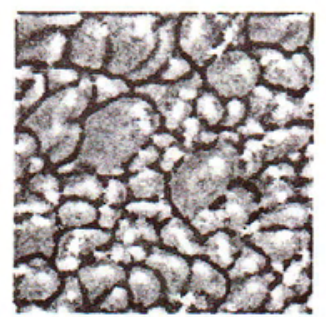

Fig. 4: Image after applying local equalization where $l$ is the given intensity, $h, v$ are given real numbers, $k$ is a sought intensity for the above-mentioned substitution, and the sum on the right hand side of the above formula is the discretized integral on the left hand side of the formula.

The result of applying local equalization is shown in Fig. 4.

\section{Technology used for acquisition of gray-scale images}

The gray-scale images (input data to the algorithm) of a grain structure are collected by STM in the case of conductive materials. In the case of different materials, a different microscope has to be used. The basic parts of STM are: a standard scanner with two piezoceramic tubes having outer and inner electrodes and with a scanning tip, and the carriage with a material sample.

The scanning of the sample surface works on the principle of tunnel current between the sample and the microscope. The voltage is fed to the carriage. The level of the tunnel current depends on the amount of voltage and on the distance between the sample surface and the scanning tip. The closer the point at the sample surface is to the scanning tip, the stronger the current. The magnitude of the current intensity is stored to the gray-scale image that serves as an input to the algorithm.

The scanned area is fixed. The voltage on the outer labels of the piezoceramic tubes of STM is controlled by the size of the scanned area.

Detailed information about the technology used and about the microscope used is presented in many journals and books, see, e.g., [7], [9], [10].

\section{Characteristics of the gray-scale images (input data)}

Technical imperfections of STM result in relatively noisy images with undesired artefacts, for example: the real grain boundaries vanish and unreal boundaries arise, and many artificial local extremes in the image are generated (because of the resulting roughness of the intensity distribution). The rough boundaries are formed particularly by breakdown vibrations of the microscope-scanning tip.

It may be assumed from reality (when ovals approximate grains) that the local intensity peaks are in accordance with the grain peaks, and the local intensity minima coincide with the grain boundaries. The intensity values between the peaks and the boundaries gradually decrease from the local intensity peaks to the local intensity minima.

The average grain size depends on the size of the scanned area. The smaller the scanned area of the sample, the bigger the grains are in the image, and there are fewer grains in the image. The larger the scanned area, the smaller the grains are in the image, and the greater the number of grains in the image. The images are distributed to the following three categories:

- Category 1 (big scanned area, small grains),

- Category 2 (the intermediate case),

- Category 3 (small scanned area, big grains). 


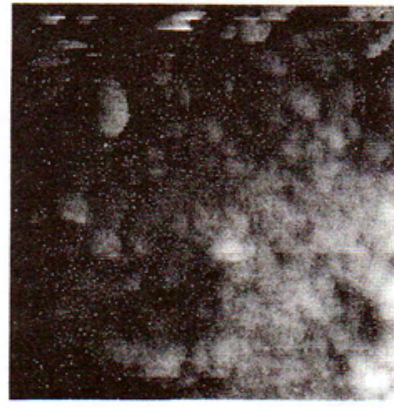

Fig. 5: Big area, small grains (Category 1)

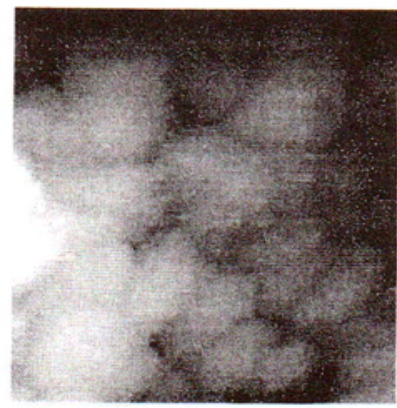

Fig. 6: Small area, big grains (Category 2)

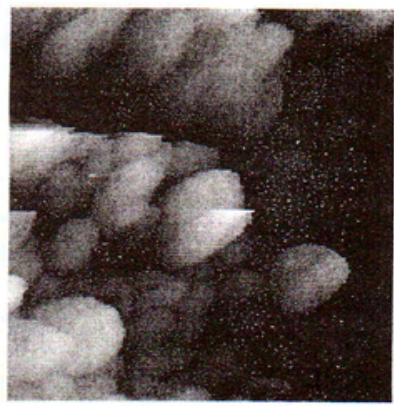

Fig. 7: Intermediate case (Category 3)
The boundaries between categories are defined experimentally, as illustrated in Fig. 5, Fig. 6, and Fig. 7.

The local average intensity near the points varies with their position in the image, and with the grain size (see Figs. 5-7).

The part of the algorithm for calculating the probability distribution of the grain size assumes that the grains in the sample have a non-ideal sphere-like shape. If this shape cannot be assumed for any reason, the method of description of the local curvature cannot be used by the procedure established in Section 6.

\section{The algorithm}

The input to the algorithm is a gray-scale image (input image), which represents the surface of a trial sample, see Fig. 8, for example.

The first step of the algorithm is to determine the parameters for the pre-processing of the input image. This involves assigning the input image to category 1, 2, or 3 - as shown and defined in the forthcoming Section 5.1.

The next step of the algorithm is to investigate the local intensity extremes of the input image and use the local in-

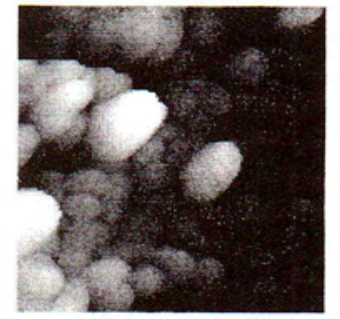

Fig. 8: View of the gray-scale input image

tensity extremes for the localization of grain boundaries. The localization begins at all local intensity peaks. Intensity minima serve as stop conditions of the localization.

The data of the grain boundaries are used for calculating the probability distribution of grain size in the final step. There are two methods defined for the calculations: the method of the two most distant points and the method of description of the local curvature of grain boundaries.

Output data with the probability distribution may be represented by a graph, or by a simple data summary.

The overview of the algorithm is shown in Fig. 9.

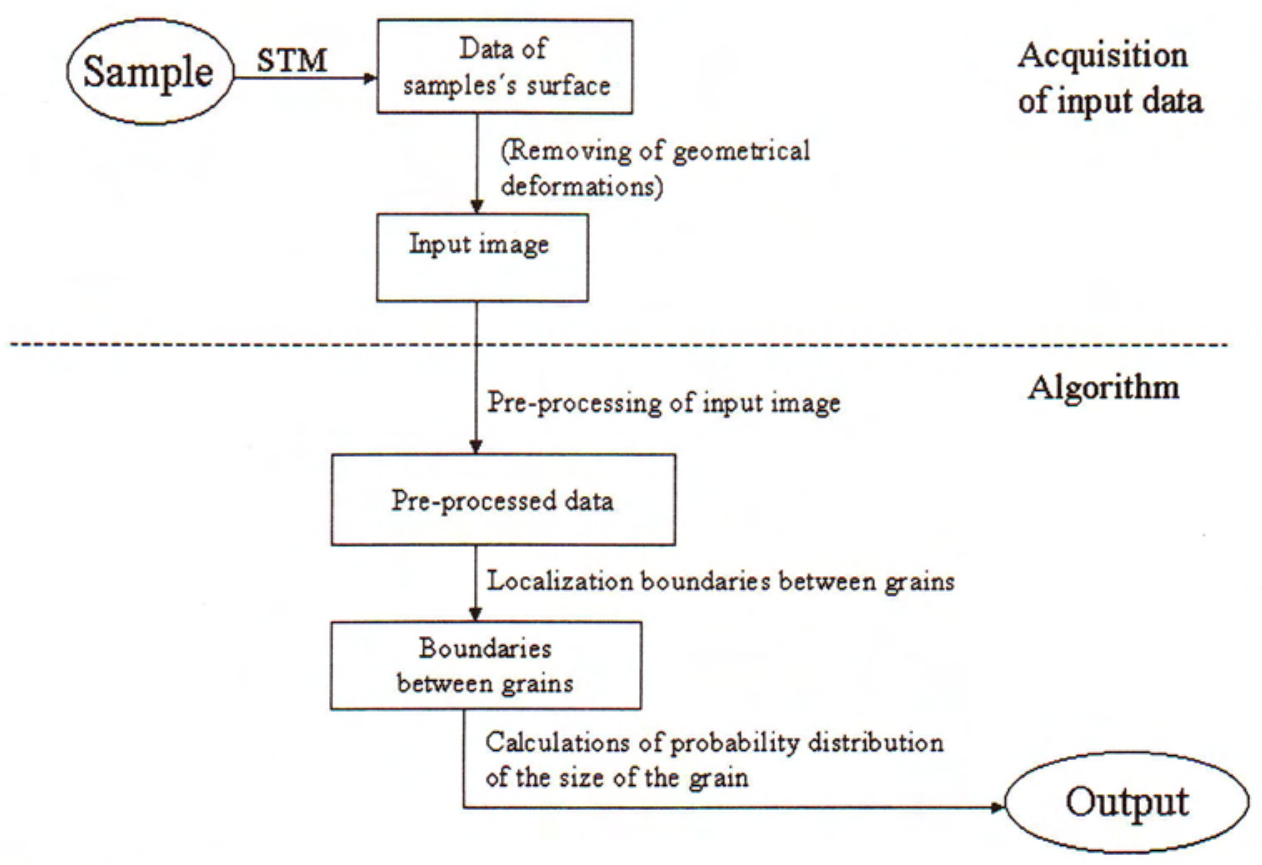

Fig. 9: Chart of the algorithm 


\subsection{Assigning the input image to a category}

The aim of this algorithm step is to match the input image to the above-defined category (recall that we consider three categories, cf. Fig. 5-7), and to select the size of the square neighborhood for subsequent use in the pre-processing of the input image and in the search for local peaks in this image. The neighborhood may also be determined manually with respect to the value entered by the user - the average size of grains in the image may be improved by hand.

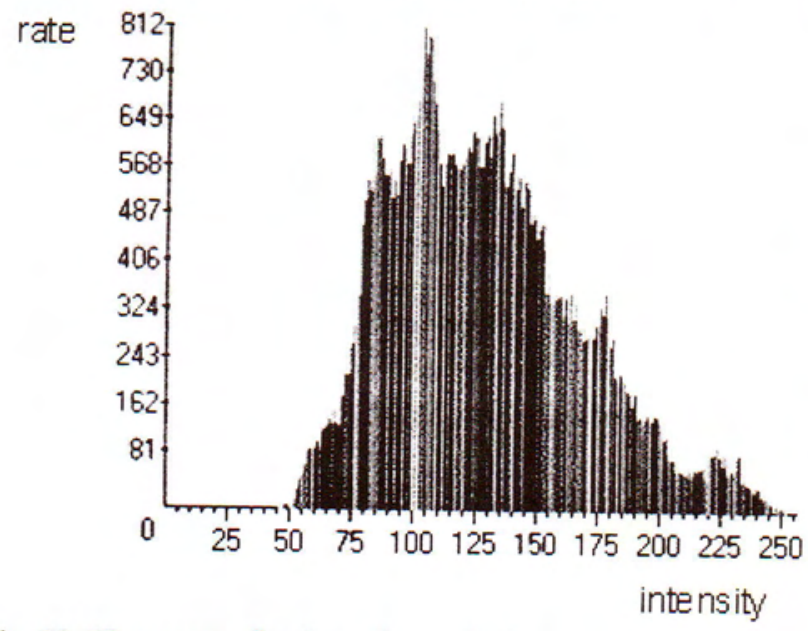

Fig. 10: Histogram of an input image in Category 1

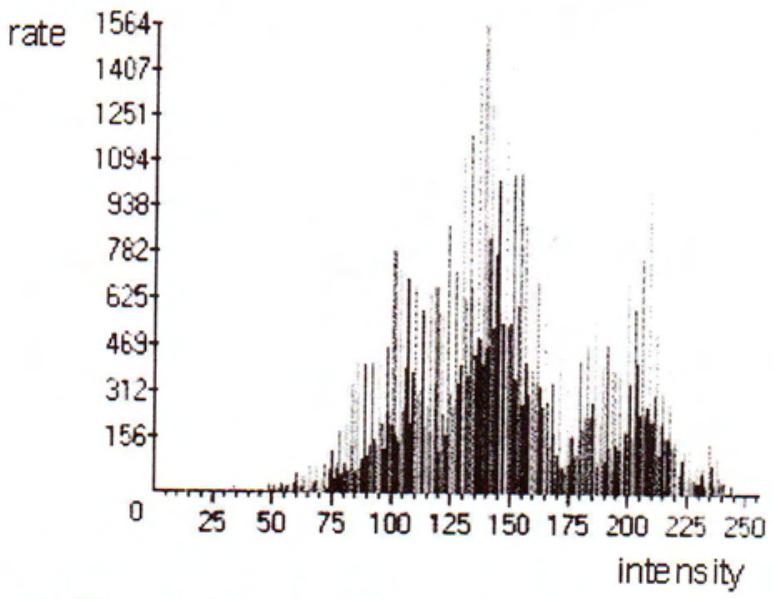

Fig. 11: Histogram of an input image in Category 3

\section{Automatic categorization}

The category is determined from the histogram of the input image after applying "local equalization" (neighborhood 7 - for more details see Section 2.3) to the input image. It has been shown experimentally that smaller grains have a smoother course in the resulting histogram. The coefficient of the smoothness of the resulting histogram is calculated as the sum of the differences of neighboring values in the histogram. The category of the image (and the necessary size of the square neighborhood used by subsequent algorithm steps) is determined.

\subsection{Pre-processing of the input image}

First, the input image is partially freed of noise by using the "square median filter" (for detailed information see Sec- tion 2.1). The image is then to some extent free of disturbed grain boundaries and free of artificially generated boundaries. It has been shown experimentally that it is more convenient to run the "square median filter" several times in a small neighborhood than only once in a larger neighborhood.

After applying the "square median filter", "local equalization" is applied to equalize the local brightness changes in the image and to unify and emphasize information about the grain boundaries and grain peaks. Before the application of "local equalization", it is possible only to claim that potential peaks of grains (as starting points for the localization of grain boundaries) are local intensity maxima. This statement is weaker than after applying "local equalization". After applying "local equalization", it can be claimed that the local intensity peaks from a certain intensity are potential grain peaks. It has been shown experimentally that "global equalization" (for more details see 2.3) applied to the image after "local equalization" delivers better results. Fig. 12 shows the image after application of the "square median filter" and "local equalization".

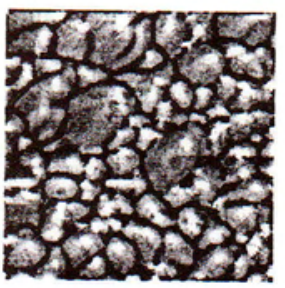

Fig. 12: A locally equalized image

Finally, the „Gaussian low frequency filter" (for more details see Section 2.2) is applied to the image to eliminate high frequencies in the image - artificial local extremes generated by erroneous vibrations of the microscope tip. The result is very close to that depicted in Fig. 11.

\subsection{Search for local intensity peaks in the image}

The application of the various filters (in the previous step) removes the noise from the image and emphasizes local extremes of the intensity function, which represents the gray-scale image.

In this step of the algorithm, local intensity peaks in the image are searched (after pre-processing of the input image). The peaks are taken as the basis of grains for segmentation of grains by the flood-growing process. Local peaks are searched in the square neighborhood of a defined size (defined within the categorization of the image).

\subsection{Search for grain boundaries in the image}

This section describes in detail the part of the algorithm for localizing grain boundaries. The transformations (of images) mentioned in this section are described in detail in Section 2. More information about algorithms for localizating boundaries between objects is presented in many books and journals, see, e.g., [1-2], [4-6], [8].

The search for boundaries consists of two steps:

First, the local intensity peaks revealed in the previous algorithm step are taken as the basis for potential grains, and the grains are segmented by the flood-growing process with 
the criterion of decreasing intensity of neighboring points. This concept is applied because the grains are approximately oval. The tops of the ovals have the highest intensity in the region of the grain (as they are closest to the microscope tip during microscope scanning). By contrast, points on the boundaries between grains have a locally minimal intensity (as they are furthest from the microscope tip).

In the second step, some areas must be linked together, as many undesirable intensity peaks could be due to artefacts that were not eliminated during pre-processing of the image. As regards artificial intensity peaks, during segmentation the grain may be divided into a large number of smaller areas (than the real region of the grain). The grains are linked with the use of experimentally defined criteria.

\section{Segmentation of grains with flood-growing}

In this step the algorithm may be summarized as follows:

1. The local intensity peaks are taken as one-point-areas of grains. These peaks have certain intensities (grainInt).

2 . The global intensity counter is initialized at the maximum intensity. The counter can be denoted as globInt (globInt $=255$ ).

3. All areas of grains are cyclically tested until at least one neighbor of the point ("trial point") in each area is found that does not yet belong to an area and which has intensity equal to the counter globInt value. Let us denote this found neighbor as the "eligible point". This "eligible point" is added to the area of the above-mentioned "trial point" and the cycle is repeated until the end of one loop. The cycle is stopped when there is no "eligible point".

4. GlobInt is decreased by one. If the globInt counter is greater than the lowest intensity 0 ( 0 is taken as the image background), the algorithm continues from step 3, otherwise the algorithm for the flood-growing process is stopped.

Now let us show that the algorithm fulfills the following:

- each point in the image is added to a grain,

- the algorithm realizes the concept of the flood-growing process of the grains from the found peaks,

- no grain overlaps another grain in the actual implementation of the algorithm described in this paper and partial areas of grains touch at the real boundaries of the grains.

Local intensity peaks are selected in the whole range of the image, so the intensities of the neighbors of the local peaks may only be lower, or the same, but cannot be higher. The points on the boundary between two grains are local minima. The points between the boundary of a grain and the peak of the grain belong to the region of the grain, and their intensities are between the intensity of the peak and the intensities of the points on the boundary. Points belonging to no area yet in the image are added to areas when they are neighbors of an existing area and their intensity is equal to or greater than the globInt counter value. The value of the globInt counter is decreased in each loop according to the steps of the algorithm. These substances demonstrate that points with equal or lower intensities than the intensities in the scope of the given grain are added to the area of the given grain and the two grains are in contact at their common intensity boundary. Thus, the areas of the grains are segmented with the flood-growing process from peaks to intensity minima in the scope of the grain with the criteria of decreasing intensity.

Now we will show that in an ideal image one area should not overlap another area. Let us take a particular grain (current grain). If the points of the neighboring grain were added (in an ideal image) to the current grain, the intensities of the attached points would not be lower than or equal to the intensities in the current grain. The function of intensity between the peak and the boundary would not decrease, but it would rise, and this fact would be contradictory to the assumptions and to the implementation of the algorithm. Another benefit for the algorithm is that the meeting of two-grain regions is at the boundary where the intensities are equal to a value of globInt. All neighboring points have a higher intensity, so that at the time when the boundary is reached, the neighboring points have been added to an area, and they cannot be added to another area (at least in an ideal image). A real input image can contain small mistakes in the course of the intensity function.

As globInt ranges from 255 to 0 and as each point in the image is tested to be a neighbor with an intensity equal to the globInt value of an existing region of a grain (in each phase of the cycle, if the point is not yet a member of a region), at last at globInt $=1$ each point (with an intensity different from 0 ) must be added to the region of the current grain. Points with zero intensity are taken for the background of the image.

Fig. 13 shows the output of this step of the algorithm.

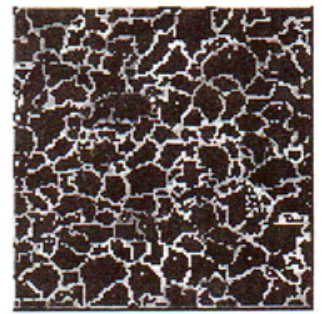

Fig. 13: Boundaries between areas (involving artificial boundaries)

\section{Linking of segments of grains (in the sense of specified criteria)}

Due to technical imperfections of the scanning microscope, there are many artificial local intensity peaks in the input image. For this reason, the algorithm finds more areas than desired (corresponding to reality). Calculations of the algorithm can divide the region of the grain into several smaller areas. From the previous text we know that in the range of one grain the average intensity of the points inside the region of the grain is greater than the average intensity on the boundary of the grain, and is lower than the intensity of the peak. Because of the assumed oval shape of the grains and the application of "local equalization" on the image it is possible to determine, from the intensities of the areas boundaries, whether the boundary between two areas is artificial. The algorithm and the criteria can be categorized as follows:

1. First, two areas are found in such a way that they comply best with the following criterion. This is the basic criterion for linking the segment areas of the grains. For each two areas, the intensities of all points on their shared bound- 
ary (on the boundary belonging to one grain and on the boundary belonging to the other grain) are received and the average of all the intensities is calculated. The two areas that have the highest average comply best with the criterion.

2. This highest average is considered and compared with the experimentally given constant limit. This limit determines the value of the average when linking two areas (with the boundary average above the limit) and not linking (with the boundary average below the limit). If the highest average is lower than this limit, the algorithm is stopped, as the other lower averages are also lower than the limit. As long as the average is greater than the limit, the algorithm passes to the next step. The determination of the limit based on experimental observations is without loss of generality, as unification (with the help of "local equalization") of important intensities in the scope of the grain does not greatly depend on the given image.

3. Finally, the auxiliary criterion for linking the segment areas of the grains is tested. The points on the common boundary of the two selected areas of the grains are considered, and the averages of the intensities of their boundary points for each area are now calculated separately. As each grain has its own boundary, the boundary between two grains is double pixeled. One boundary belongs to one grain and the second to the other grain. In section 1 , the average is calculated together. The two averages are compared. If the values of the averages are similar, the two areas are linked to a single area and the algorithm returns to section 1 . If the values of the averages are different, "local equalization" fails and these areas should not be linked. The fact that these two areas are not to be selected in the following stages is marked in the memory, and the algorithm returns to section 1 .

Fig. 14 is a sketch of the final grain boundaries.

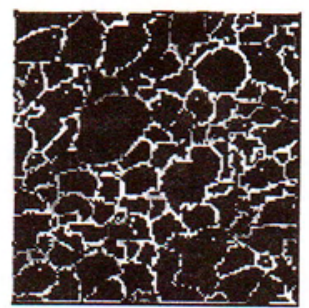

Fig. 14: Boundaries between areas (not involving artificial boundaries)

\section{Calculation of the probability distribution of grain size}

Data on the grain boundaries are used for calculations of the probability distribution of grain size. Two methods are suggested and implemented for implementing these calculations:

- method of description of the local curvature of grain boundaries,

- method of the two most distant points.

The results of these calculations may be illustrated in the form of a graph or written in standard output.

\subsection{Method of description of the local curvature of grain boundaries}

This method assumes that grains can be approximated by spheres. In this method, the size of a grain is measured by its radius.

If this assumption is fulfilled, we can suppose that in vertical scanning of sample surfaces, the resulting boundaries are almost circular in shape. If the grains overlap, the resulting boundaries are composed of concave and convex curves and form a closed curve (at least in the case of an ideal image). The concave parts of the curves are formed by cuts of the mutual overlaps. Consequently, only the points of the convex parts of the boundaries are considered for calculations of the radius of the sphere grain. These are points on the real surface of the grain, and not points generated by overlapping.

\section{Theory for calculating the radius of a sphere (circle)}

If the boundaries between grains are approximated by an ideal circle (with radius $r$ ), then the points of this circle can be parametrically described by the following function:

$$
k(t)=[x(t), y(t)],
$$

where $t$ is the parameter of 0 to $2 \pi r$ and

$$
x(t)=r \cdot \cos (t / r), y(t)=r \cdot \sin (t / r) .
$$

Curvature curv at the point of function $f(t)=[x(t), y(t)]$ is defined as:

$$
\operatorname{curv}[f(t)]=\frac{\partial^{2} f}{\partial t^{2}}(t)=\left[\frac{\partial^{2} x}{\partial t^{2}}(t), \frac{\partial^{2} y}{\partial t^{2}}(t)\right] .
$$

Curvature at the point of the circle after substitution of $k(t)$ for $f(t)$ is given by:

$$
\operatorname{curv}[k(t)]=\left[-\frac{1}{r^{2}} \cdot \sin \left(\frac{t}{r}\right),-\frac{1}{r^{2}} \cdot \cos \left(\frac{t}{r}\right)\right] .
$$

The extent of the curvature at points $k(t)$ for $t=0$ to $2 \pi r$ is:

$$
|\operatorname{curv}[k(t)]|=\sqrt{\frac{1}{r}}, \text { so } r=\sqrt{\frac{1}{\operatorname{curv}[k(t)]}} .
$$

\section{Algorithm for calculating the radius of a sphere (circle)}

For each point on the boundary of a grain the following steps are carried out:

1. Differentiating the boundary curve twice, the resulting gradients are used for determining whether the trial point is in the convex or the concave part of the boundary curve.

2. If the point is in the convex part of the boundary curve then the curvature value is calculated. The curvature value is calculated with the use of numerical methods based on $n$ neighboring points (Lagrange, Tschebysheff). The radius then follows from the above-defined formula and is stored in an auxiliary buffer.

The most extensive value of the radii is calculated from the auxiliary buffer. The most extensive value is declared as a resulting radius of the grain and put into the resulting graph of the probability distribution of grain size.

Remarks on implementation: It is necessary to smooth the boundary curve of the grain before calculating the local curvatures, as calculations of the local curvature are based on the second differentiation (using $n$ neighboring points), which are numerically ill posed. 


\section{Smoothing}

The parametric curve is determined in $n$-dimensional space by the formula for $x(t)=\left[x_{1}(t), \ldots, x_{n}(t)\right]$, where $t$ is a parameter of the length of the curve. The principle of smoothing involves multiplying the second derivative at the trial point $x(t)$ by parameter $l$ and adding the result to the point itself:

$$
\frac{\partial^{2} x}{\partial t^{2}}(t) \cdot l=\left[\frac{\partial^{2} x_{1}}{\partial t^{2}}(t) \cdot l, \ldots, \frac{\partial^{2} x_{n}}{\partial t^{2}}(t) \cdot l\right],
$$

where $l$ is entered as a very small number (0.01).

\section{Evaluation of this method}

This method provides good results on ideally generated circles, but in practice, in many cases, the results are worse. The input images are often very noisy and calculations of the second derivative fail, i.e., they are numerically ill posed.

\subsection{Method of the two most distant points}

This method consists in determining the radius of a grain as the maximum of the distances between each two points in the domain of the grain. The maximum distance is stored into the probability distribution graph of grain size. If the grains are approximated by spheres (circles), the distance is divided by two to get the sought radius.

\section{Evaluation of this method}

This method provides good results in practice. It may fail only when the grains overlap excessively. This is not a frequent case, especially because of the assumption that the grain surfaces are spherical.

\section{Results of tests}

To test the algorithm, a $\mathrm{C}++$ program has been created. Images with the grain structure of the surface of gold samples are used as input images for the algorithm. The generation of ideal circles is also inserted to the program. When implementing the algorithm, emphasis is put on the functionality of the program and on speed of calculation. The processing of one image takes about 20 seconds, and the boundaries between grains are found to be almost correct. Worse results are obtained when calculating the probability distribution grain size. The tests were performed with approximately 100 different images.

In the final evaluation attention should be paid mainly to the following stages of the algorithm:

- categorization of the input images,

- pre-processing of the input images,

- search for grain boundaries,

- calculation of probability distribution of grain size.

As the input images are very different, they are distributed to the three categories (see Section 4). Each category is assigned coefficients for pre-processing the input image, local transformations and searching for the grain boundaries. The input image can be taken back to one of the categories manually by the user or automatically by the algorithm.

Pre-processing of the input images is performed by means of standard transformations. In this stage of the algorithm, emphasis is put on enhancing the quality of the input images. The input images are very noisy and include many artificial artefacts. Without this stage, the algorithm would perhaps fail.

The stage of searching boundaries between grains works very well, even with very poor quality input images. The boundaries correspond to the real boundaries that can be seen. This is good for the algorithm because the following stage of calculating the probability distribution is very dependent on the results of this stage, especially in the case of the method of description of the local curvature.

The graph of the probability distribution of grain size given by the method of the two most distant points is shown in Fig. 16. Fig. 17 is the same graph, for the method of description of the local curvature. The input image for the algorithm is taken from Fig. 15 with ideal circles. Fig. 16 and Fig. 17 show that the method of two most distant points

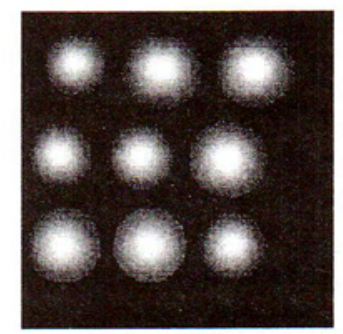

Fig. 15: Image with ideal circles (radius 25 pixels)

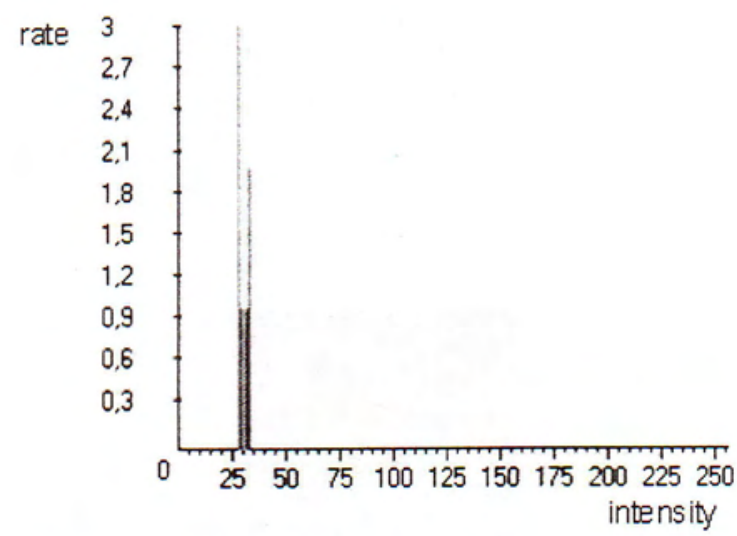

Fig. 16: Method of the two most distant points

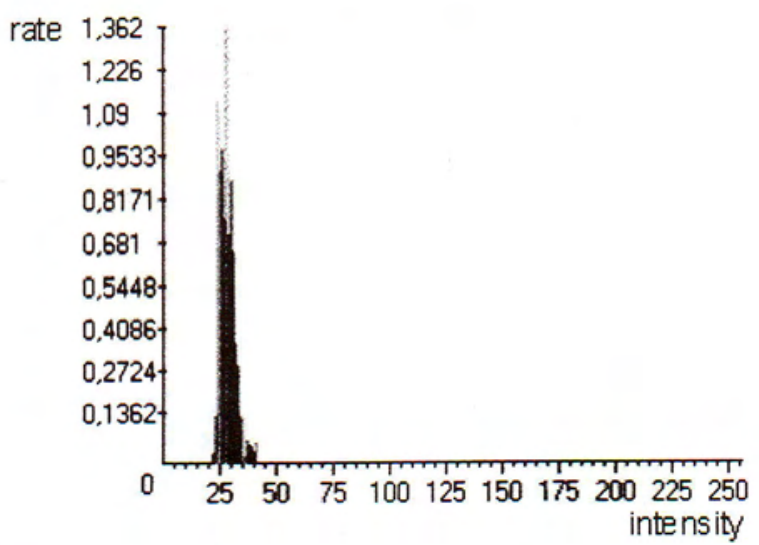

Fig. 17: Method of description of the local curvature 
delivers better results than the method of description of the local curvature. The rate of dispersion of the radii is obviously larger in the second figure. This is because the input images have very poor quality and the calculations of the second derivatives (necessary for calculating the local curvatures) are numerically ill posed. The method of description of the local curvature has its use mainly when calculating the distribution probability of "ideal spheres" sizes. It can be used, e.g., in programs manipulating ideal shapes (circles, spheres).

\section{Other possible solutions}

Other possible methods for localizing grain boundaries may include edge detectors or morphological operations. Experience acquired while implementing the algorithm shows that these methods (based on edge detectors or morphological operations) would probably be less correct, and they would be more difficult to implement.

\section{Conclusions}

In this paper, the results of scanning of the surfaces of structural elements at nano-level are improved using mathematical approaches. Among these approaches belongs smoothening of the scanned surfaces and searching for boundaries or contour curves being related to some levels. The peaks of the surfaces are also determined. Accuracy study is carried out for couple of samples. Different procedures suggested provide reasonable results in the case of different samples.

\section{Acknowledgment}

This paper was financially supported by GAČR, project number 103/03/1083.

\section{References}

[1] Boyle, R., Hall, Ch., Šonka, M., Hlaváč, V.: Image Processing, Analysis and Machine Vision. PWS Publishing, Pacific Grove or Cambridge Publishing House, 1999.

[2] Pratt, W. K.: Digital Image Processing. Second Edition. A Wiley-Interscience publication, 1991.
[3] Jarkrans, T.: Algorithm for Cell Image Analysis in Cytology and Pathology - Dissertation theses. ACTA UNIVERSITATIS UPSALIENSIS UPPSALA, 1996.

[4] Bamford, P., Lovell, B.: A Water Immersion Algorithm for Cytological Image Segmentation. APRS Image Segmentation Workshop, University of Technology Sydney, December 1996, p. 75-79.

[5] Bamford, P., Lovell, B.: Improving the Robustness of Cell Nucleus Segmentation. Proc. British Machine Vision Conf., BMVC'98, England (UK): University of Southampton, September 1998, p. 518-524.

[6] Bengtsson, E.: The Measuring of Cell Features. Anal. Quant. Cytol., Vol. 9, (June 1987), No. 3, p. 212-217.

[7] Maurel, C., Coratger, R., Ajustron, F., Seine, G., Pchou, R., Beauvillain, J.: Effect of Multiple Tips on Light Emission Induced by STM from Gold Nanostructures. Toulouse (France): Centre d'Elaboration des Materiaux et d'Etudes Structurales-CNRS.

[8] Brandtberg, T.: Towards Structure-based Classification of Tree Crowns in High Spatial Resolution Aerial Images by Multiple-scale Analysis. Scand J, Forest Res 12, p. 89-96.

[9] Hwang, I. S., Lo, R. L., Tsong, T. T.: J. Vac. Sci. Technol. Al6 4, 1998, p. 2632-2641.

[10] Durand, H.-A., Sekine, K., Etoh, K., Ito, K., Kataoka, I.: 1998 Int. Conf. on Ion Implant. Tech. Proc., IEEE, NJ, 1999, p. 929-935.

Prof. Ing. RNDr. Petr P. Procházka, DrSc.

phone: +420224354480

fax: +420224310775

e-mail:petrp@fsv.cvut.cz

Mgr. Michal Toman

Dept. of Structural Mechanics

Czech Technical University in Prague

Faculty of Civil Engineering

Thákurova 7

16629 Prague 6, Czech Republic 